\title{
Design and implementation of control Cabinet Based Siemens S7-300 Programmable Logic Controller PLC
}

\author{
Basim Mohsin Abdulwahid Al-Najari \\ \{pe21023@student.uniten.edu.my\} \\ University Tenaga Nasional UNITEN, Malaysia
}

\begin{abstract}
The present proposal is to design and implement of control cabinet-based Siemens S7-300 programmable logic controller (PLC). Using PLC in industries is easy, resilient, and more compact than the classic system. The cabinet will be used in both training and research activities. It consists of two parts hardware and software. The hardware consists of several cards such as power supply, Central Processing Unit (CPU), digital input, digital output, analog input, and analog output. On the other hand, the software is the Totally Integration Automation (TIA) and it will be installed on a Personal Computer (PC). The TIA is the latest version of Siemens software, and it consists of two parts, logic builder (Step7) and Human Machine Interface (HMI) builder. In TIA, the logical diagram and HMI pages of the process can be designed to fabricate supervisory control and data acquisition (SCADA). An applied program will be written and loaded in the PLC to test the overall system.
\end{abstract}

Keywords: PLC, SCADA, CABINET, TIA, HMI, CPU, CCR.

\section{Introduction}

Programmable logic controllers (PLCs) have been used in industry in one form or another for the past twenty years. The PLC is designed as a replacement for the hardwired relay and timer logic to be found in traditional control panels [1]. In today's world, many industries are getting automated using PLC and thus it has become the main part of the industries [2]. It acts a major function in the automation field which tends to reduce complexity, increases safety, and be cost-efficient [3]. Input devices: for example, switches, and output devices, for example, motors, being controlled are connected to the PLC and then the controller monitors the inputs and outputs according to this program stored in the PLC [4]. In this paper, a programmable logic controller experiment set for education was designed [5]. These controllers are specially designed to survive in harsh situations and shielded from heat, cold, dust, and moisture, etc. PLC consists of a microprocessor that is programmed using the computer language. The program is written on a computer and is downloaded to the PLC via cable [6]. Automation is the delegation of human control functions to technical equipment [7]. This Laboratory Trainer and research setup system is fully integrated with PLC Motion Animation software. The software tool can be used as an offline and Online animation tool with its collection of sample HMI applications. The trainer can be used alternately for the operation and control of industrial manufacturing processes [8]. The PLCs consist of power supply, Central Processing Unit CPU, memory, and input \& output modules. The input unit receives the field signals from the input devices and converts these signals to a form compatible with the CPU requirements. The output 
unit (a set of relays, transistors, or Triac) translates the CPU orders to field actuators. Memory is used to save the user program, data, and configuration [9]. The advance of technology and electric drives raises the usage of PLC in industrial systems. The use of Programmable Controllers in automation things increases flexibility and reliability of processes and expands and enhances the production [10]. Wireless technologies are rapidly being adapted from simple monitoring and control to supervisory control and data acquisition (SCADA) systems. Wireless provides highly reliable data communications in a harsh and interference-heavy environment [11]. Recent developments in Human Machine Interface (HMI) have brought remarkable advances in performance, flexibility, and openness. Selecting an HMI interface for a PLC-based automation system that will meet the needs of the process over the projected lifetime of the control system requires consideration of many issues [12]. Hence it has become difficult to debug the failures in the system and it is a highly feasible job to facilitate alternate connections to the fault-tolerant systems in the relay logic [13]. In this task, the analytical compute model for optimizing the functions of the volume processing plant can handle all possible decision variables in the setup [14]. For complete control of the building, a proper SCADA implementation and optimization strategy must build [15]. This paper presents the design and implementation of control cabinet-based Siemens S7-300 PLC.

\section{Cabinet Implementation}

The Siemens PLC cabinet consists of two parts as below:

\subsection{Hardware}

\subsubsection{Enclosure}

The enclosure is used to contain the electrical modules and their connections to fabricate Siemens PLC rack type and it is $70 \mathrm{~cm} * 50 \mathrm{~cm} * 20 \mathrm{~cm}$ dimensions.

\subsubsection{Power Supply}

The power supply is used to supply the voltage to the CPU and input-output modules. The type of this supply is Siemens PS 307 with output current equal to 5A, input voltage equal to (220) VAC, and output voltage equal to 24VDC. Fig 1 . shows the wiring connection of this module.

\subsubsection{Central Processing Unit CPU Module}

The central processing unit (CPU) is the main part of the cabinet. it is modular and compact, versatile, a secure investment, and is powerfully fit for a full range of applications. A scalable and flexible design, a communication interface that fulfils the highest standards of industrial communication and a full range of powerful integrated technology functions make this CPU an integral part of a complete and comprehensive automation solution. It is standalone or dedicated to run one program that continuously monitors the state of the input devices and makes decisions based upon a custom program to manipulate logically the state of the output devices. The CPU that is used in this research is Siemens S7-300 model CPU315 2pn/dp. Fig 2. shows the CPU wiring connections. 


\subsubsection{Micro Memory Card (MMC)}

The micro memory module is a permanent storage media. it is used as a part of PLC to store and backup programs and data without battery. The PLC is a battery-less device, and it loses its program in case of power off. When the PLC starts, it loads the user program from the MMC and stores it in its internal memory (RAM). The PLC is not operating without MMC. The type of MMC in this research is a FLASH EPROM and Its capacity is $512 \mathrm{~Kb}$.

\subsubsection{Digital Input Module}

The digital input module is used to input the safety devices signals such as pressure switch, level switch, flow switch, temperature switch, limit switch .ext. the model of this module is SIMATIC SM 321 DI32*DC24V. The number of input channels is (32) channel (from I0.0 to I3.7) and the signal level per channel is $24 \mathrm{vdc}$. Sixteen (16) Snap switches are used to test the module inputs. Fig 3. shows the wiring connection of this module.

\subsubsection{Analog Input Module}

The analog input module is used to convert the analog input signal to digital or binary to be suitable for manipulation inside PLC. several types of signals can be measured through this module depends on their configuration. the signals can be bipolar voltage or current, unipolar voltage or current signal, Temperature or Resistance. The process signals that can be measured by this module such as pressure, level, flow, temperature, vibration, speed, weight, and so on. Table 1 shows the analog input signal and its limits. the model of this module is SIMATIC SM 331 AI $8 * 13$ bit. The number of input channels is 8 channels, and the resolution of each channel is 13 bits. Table(4) shows the channel number and its address. Mill ampere supply is used to input analog input signal (4 to 20) $\mathrm{mA}$ to test the module in the laboratory. Fig 5. shows the wiring connection of this module.

\section{A. Current-DATA Relation}

If we suppose that the input signal is a unipolar current signal with a 4 to $20 \mathrm{~mA}$ range.

From table (1) we see that when the input signal is $4 \mathrm{~mA}$, the data is 0 and when the input signal is $20 \mathrm{~mA}$, the data is 27648 therefore the relationship between the current and the DATA is linear and can be represented by the equation below:

$\mathrm{D}=(27648 / 16) *(\mathrm{I}-4)$

Where:

D: DATA.

I: current.

mA: mill Ampere.

Fig 6. represents the relationship between current and data.

\section{B. Resolution}

The resolution is the value of the input that changes the DATA one BIT.

It is a ratio between changing in current and changing in data and equal $\Delta \mathrm{i} / \Delta \mathrm{d}=16 / 27648=578.7 \mu \mathrm{A} / \mathrm{bit}$

That means $578.7 \mu \mathrm{A}$ current value changes the DATA one BIT. 
$\Delta \mathrm{i}$ : changing in current.

$\Delta \mathrm{d}$ : changing in data.

$\mu \mathrm{A}$ : micro-Ampere.

bit: is the smallest cell in a digital system.

\subsubsection{Analog Output Module}

The analog input module is used to convert the digital or binary signal inside PLC to analog value to control the end device. two types of signals output from this module depends on their configuration. the signals can be bipolar voltage or current, unipolar voltage, or current signal. Table 2 shows the analog output signal and its limits. the model of this module is SIMATIC SM 332 AO8*12bit. The number of output channels is 8 channels, and the resolution of each channel is 12 bits. Table 5 shows the channel number and its address. Mill Ampere meter is used to read analog output current (4 to 20) mA to test the module in the laboratory. Fig 7. shows the wiring connection of this module.

\section{A. Current-DATA Relation}

If we suppose that the output signal is a unipolar current signal with a 4 to $20 \mathrm{~mA}$ range. From Table 2 we see that when the DATA is 0 , the output signal is $4 \mathrm{~mA}$ and when the DATA is 27648 , the output signal is $20 \mathrm{~mA}$ therefore the relationship between the DATA and the current is linear and can be represented by the equation below:

$\mathrm{I}=(16 / 27648) * \mathrm{D}+4$

Where:

I: DATA.

D: current.

mA: Mill Ampere.

Fig 8. represents the relationship between data and current.

\section{B. Resolution}

The resolution is the value of DATA or numbers of bits that changes the output current one micro-Ampere $(\mu \mathrm{A})$.

It is a ratio between changing in data and changing in current and equal $\Delta \mathrm{d} / \Delta \mathrm{i}=27648 / 16=1.728$ $\mathrm{bit} / \mu \mathrm{A}$.

That means 1.728-bit data value changes the current one micro-Ampere.

$\Delta \mathrm{d}$ : changing in data.

$\Delta \mathrm{i}$ : changing in current.

$\mu \mathrm{A}$ : micro-Ampere.

bit: is the smallest cell in the digital system.

\subsubsection{Ethernet Cable}

Ethernet cable is used for PC-PLC Profinet data communications. It is preassembled with two RJ45 connectors (10/100MB) and with (2) meter length. Download in the PLC and upload from the PLC instructions for programming and configuration can be done by using this cable.

\subsubsection{Personal Computer (PC)}


Personal Computer PC is used as a Siemens programmer (PG) for programming and configuration. In addition, it is used as a Siemens PC station for HMI page design and display. The Totally Integration Automation (TIA) portal is installed on the PC to do all SCADA requirements. The connection between PC and PLC is a Profinet communication using Ethernet cable.

\subsubsection{Rail}

Rail is used to containing SIMATIC S7-300 modules. it is $480 \mathrm{~mm}$ in length. All S7-300 modules are mounted onto the rail. the module arrangement in the rail is according to the slot numbers. each module can be inserted in and removed from the rail easily. Table 3 shows the SIMATIC S7-300 module's horizontal arrangement in the rail.

\subsection{Software}

The software which is used in this research is the Integration Automation TIA portal V13 and it consists of two parts as below:

\subsubsection{Logic Builder}

The logic builder is the SIMATIC STEP7 V13. It is used to build the sequence of the process as instructions using ladder diagram language and store it in the PLC memory. When the PLC is in RUN mode, the sequence of the process stored in the PLC memory is executed to operate the process. The SIMATIC STEP7 V13 contains many instructions to fulfil all logical processes, mathematical processes, and batch functions.

\subsubsection{HMI Builder}

The HMI builder is the WinCC Advanced RT V13. It is used to build the HMI pages for the operator and store them on the PC. The HMI pages relate to the sequence of the process under the TIA portal environment to fabricate the SCADA system. the WinCC Advanced RT V13 contains many instructions to display the process on the computer screen. There are three types of HMI pages:

1. A graphic page is used to show the location of the device on the site and its current readings.

2. The alarm page is used to display the event's current state for each device on the site and to give an alert in case of abnormal states.

3. The trending page is used to display the signal's current values and their curves for the period such as (5 min,15 min,30 hours, and so on) according to the operator requirements.

\section{Overall System}

Fig 9. shows the block diagram of the overall system.

Fig 10. shows a photograph of the Siemens S7-300 cabinet front.

Fig 11. shows a photograph of the Siemens S7-300 cabinet inside. 


\section{Case Study}

For commissioning the hardware and software, the practical example (Level measurement and monitoring) was designed and connected to the site to test the overall system in both hardware and software.

\subsection{Level measurement and Monitoring}

The case study is to measure the level of water vessel (V-604) with (4 meters) height and (20 meters) diameter in the water treatment unit of the factory (Fertilizer Plant). The old measuring and monitoring system consists of an electronic differential pressure transmitter as a level transmitter (LT-604) and level indicator (LIA-604) to display the level value in the central control room (CCR) for monitoring and to give alarm in case of low level. Fig 12. shows the water vessel. Fig 13. shows the block diagram of the old level measuring and monitoring system. Fig 14. shows the level transmitter in the site. Fig 15. shows the level indicator (LIA 604) in the central control room CCR. The cabinet was connected instead of a level indicator to display the level of the vessel on the computer screen in the central control room CCR. Two wires of the transmitter were connected to the cabinet for reading the analog input signal on pin 2 of the module. The channel address is PIW256. Fig 16. shows the wiring diagram of the connection.

\subsubsection{Level Calculations}

For open tank calculations, the range of the transmitter can be calculated as below:

$\mathrm{p}=\mathrm{h} * \mathrm{~s} . \mathrm{g}$.

s.g=density of object/density of water.

the density of water $=1000 \mathrm{~kg} / \mathrm{m}^{3}$

the s.g of water=density of water/density of water $=1$, then

$\mathrm{p}=\mathrm{h}$

$\Delta \mathrm{p}=\mathrm{Hp}$-Lp ---------- the differential pressure=high pressure-low pressure

But $\mathrm{Lp}=0$ then

$\mathrm{Hp}=$ head

Head $=$ max - min

$\Delta \mathrm{p}=$ head

the head $=3$ meters

$\Delta \mathrm{p}=3 \mathrm{mH}_{2} \mathrm{O} * 1000$

$\Delta \mathrm{p}=3000 \mathrm{mmH}_{2} \mathrm{O}$

Then the transmitter range is from 0 to $3000 \mathrm{mmH}_{2} \mathrm{O}$.

Where the range ( 0 to $3000 \mathrm{mmH} 2 \mathrm{O}$ ) gives ( 4 to $20 \mathrm{~mA}$ ) to the analog input module in the cabinet.

Where:

p: pressure

h: head

s.g: specific gravity.

Hp: high-pressure side.

Lp: low-pressure side.

$\Delta \mathrm{p}$ : differential pressure. 


\subsubsection{The Cabinet Commissioning}

The cabinet was tested in the site (water treatment unit) for commissioning and the steps below were done:

1. The transmitter related to the cabinet, and this led to display the level of the vessel and equal to $(78.8 \%)$ on the HMI pages.

2. The low-level set was entered in the HMI graphic page and equal to (70\%).

3. The pump P-604 was started to decrease the level of the vessel and this led to minimizing the level of the vessel gradually.

4. When the level of the vessel reached less than the set (70\%), the low-level alarm displayed on the HMI pages (graphic page and alarm page) informed the operator in the CCR about this abnormal state.

5. The pump P604 was stopped, and the valve was opened to maintain the standard level of the vessel.

\subsection{Flowchart}

A flowchart is a formalized graphic representation of a logic sequence. The purpose of a flow chart is to provide programmers with a common language or reference point when dealing with a process. The flowchart of the case study is read the level value through the level transmitter (LT 604), if the level value is less than $70 \%$ alarm will be displayed on the graphic page and alarm page for operators in the CCR, then the level value will be displayed on the pages of the HMI. Fig 17. shows the flowchart of the level measurement and monitoring system.

\subsection{Ladder Diagram}

The ladder is a diagram that shows actual component symbols and the basic wiring configuration of a relay logic circuit. The Ladder diagram in the case study is consist of always false and always true internal contacts, Scale function block to scale the transmitter analog input signal 4 20mA to 0 100\% range, and the less than compare function to display the alarm on the cabinet lamp in case of level value less than $70 \%$. Fig 18. shows the Ladder diagram of the level measurement and monitoring system.

\subsection{HMI Pages}

Three pages were designed as HMI pages for operators. Three command blocks are designed to move from one page to another. The HMI pages are as below:

\subsubsection{Graphic Page}

A graphic page was designed for level measurement and monitoring to display devices and their readings such as vessel and transmitter. The alarm object flickers in red colour in case of a level value less than $70 \%$. Fig 19. shows the graphic page of the level measurement and monitoring system. 


\subsubsection{Trend Page}

The trending page was designed for level measurement and monitoring to display the response of the level transmitter for $(15 \mathrm{~min})$ in addition to displaying the current value. Fig 20. shows the graphic page of the level measurement and monitoring system.

\subsection{Alarm Page}

The alarm page was designed for level measurement and monitoring to display the low-level alarm Tag, Date of alarm, Time of alarm, and its Status. Fig 21. shows the alarm page of the level measurement and monitoring system.

\subsection{Profnet Topology (Network)}

Profinet (Process Field Net) is an industry technical standard for data communication over Industrial Ethernet (IE), designed for collecting data and controlling, equipment in industrial systems. the network in the case study consists of two devices (PLC and PC) that were connected through (2) meters Ethernet cable. each device has an internet protocol (IP). Table 6 shows the device's internet protocols (IPs). Fig 22. shows the Profinet Topology (network) of the level measurement and monitoring system.

\subsection{Cabinet Photographs}

Fig 23. shows the photograph of the overall measurement and monitoring system.

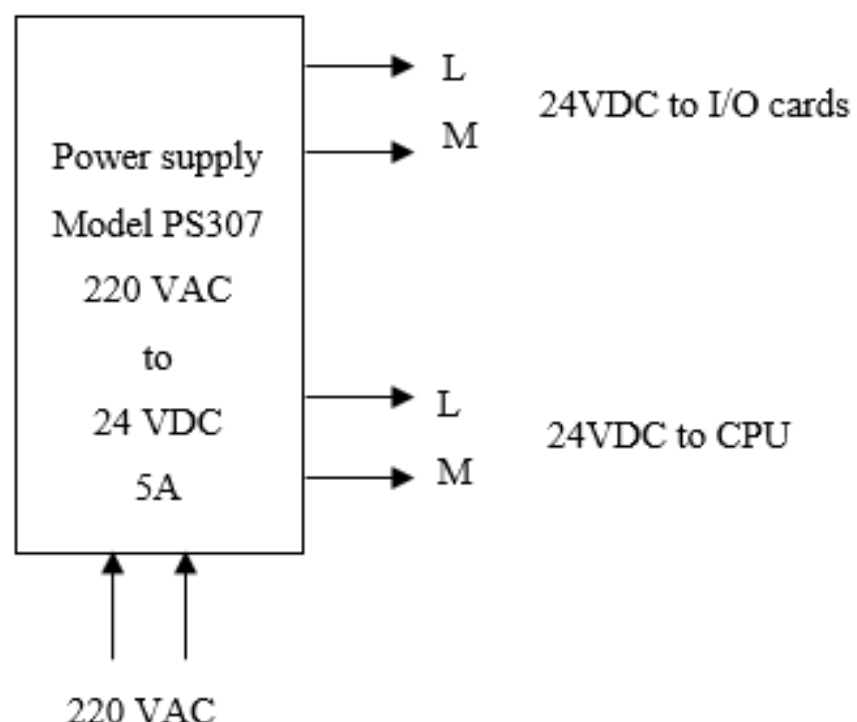

Fig 1. Power Supply. 


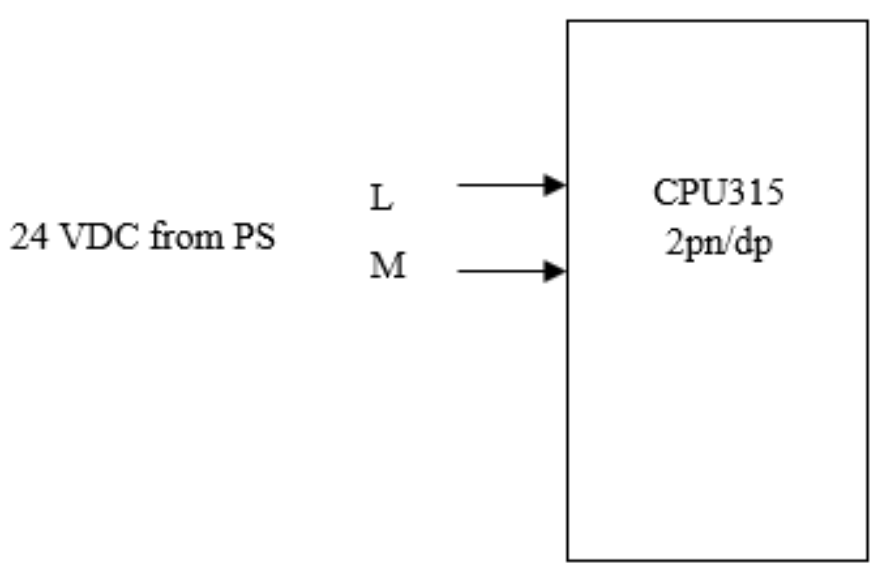

Fig 2. Central Processing Unit CPU.

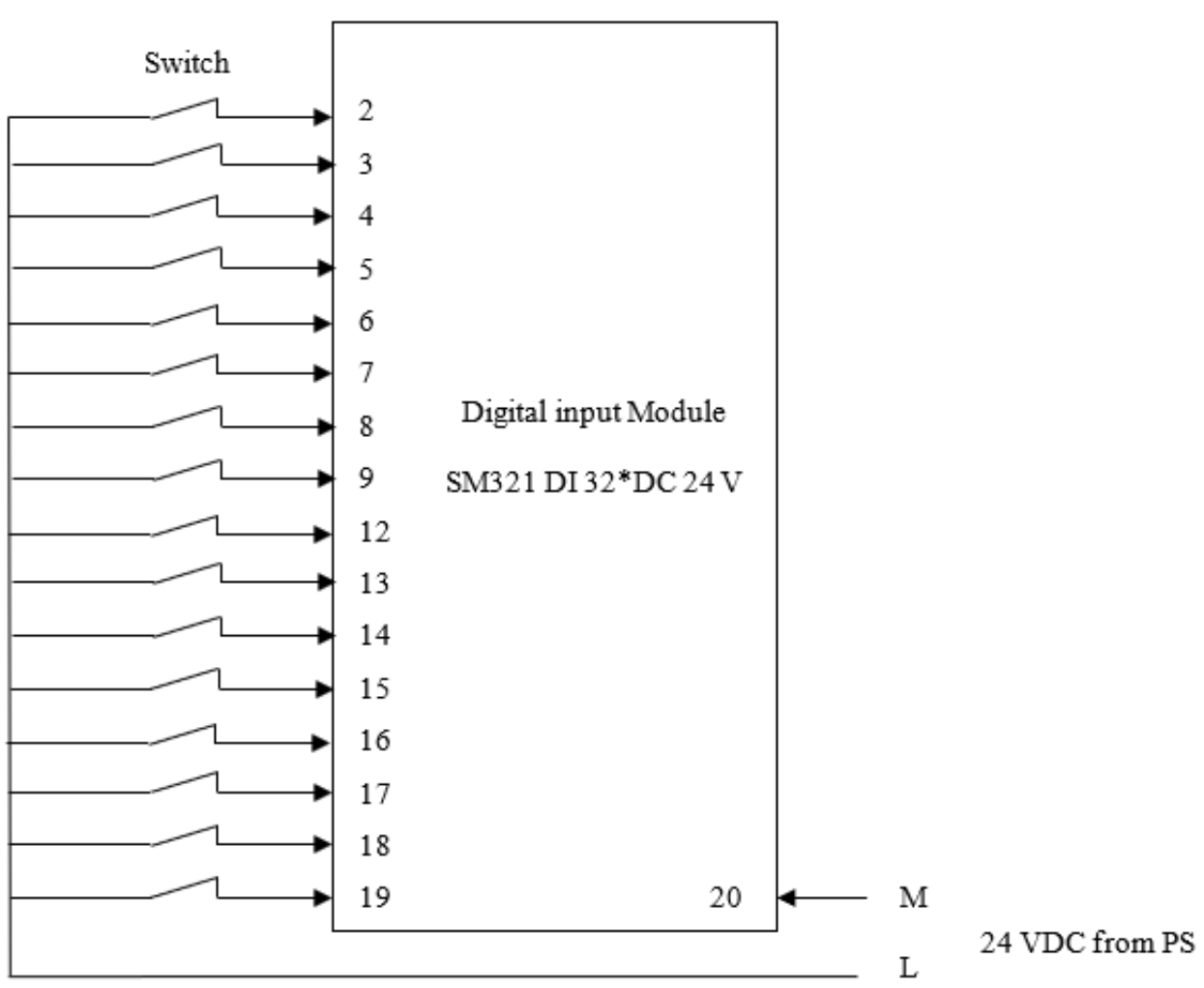

Fig 3. Digital input module. $M=$ negative, $L=$ positive. 


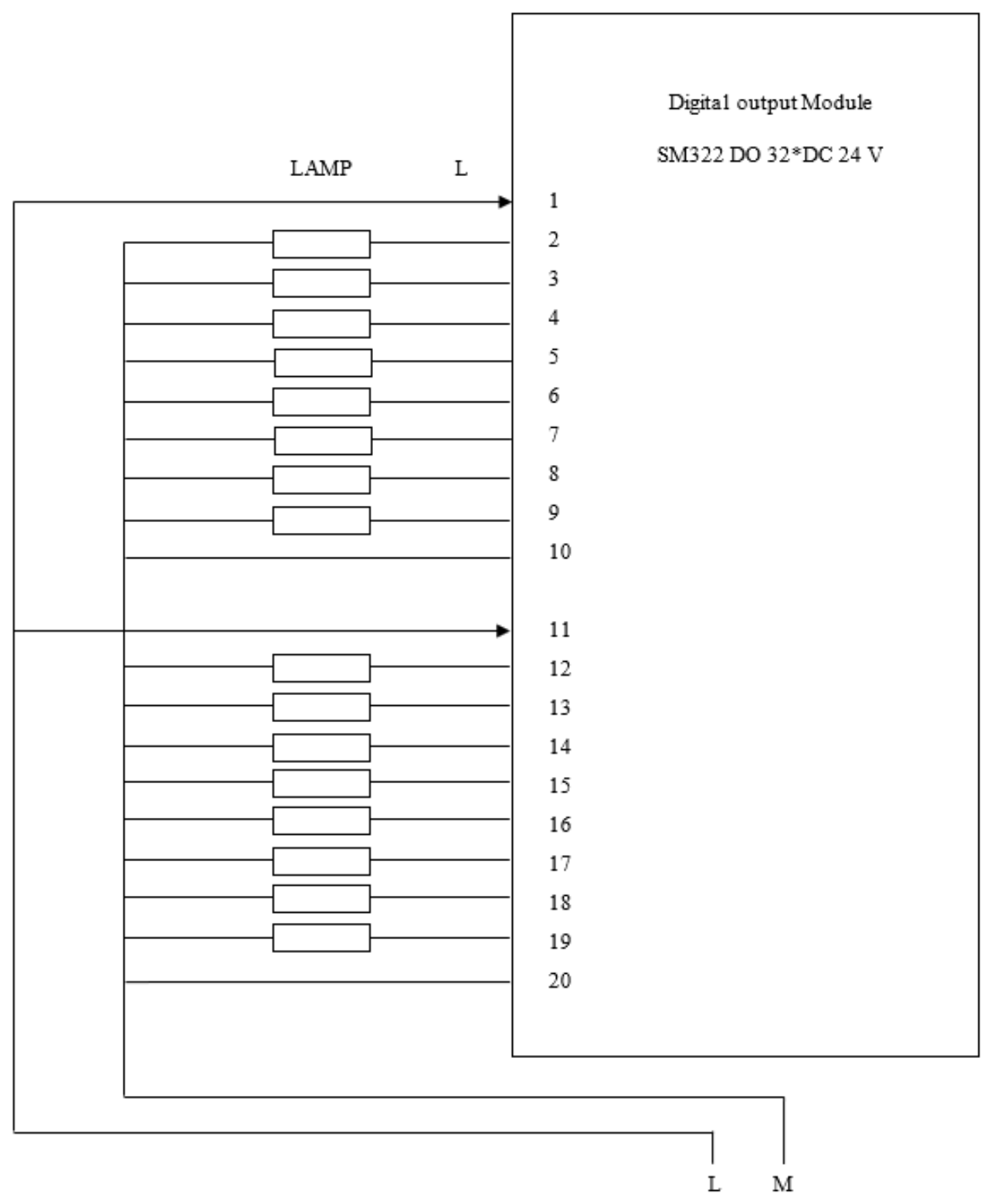

$24 \mathrm{VDC}$ from PS

Fig 4. Digital output module, $M=$ negative, $L=$ positive . 


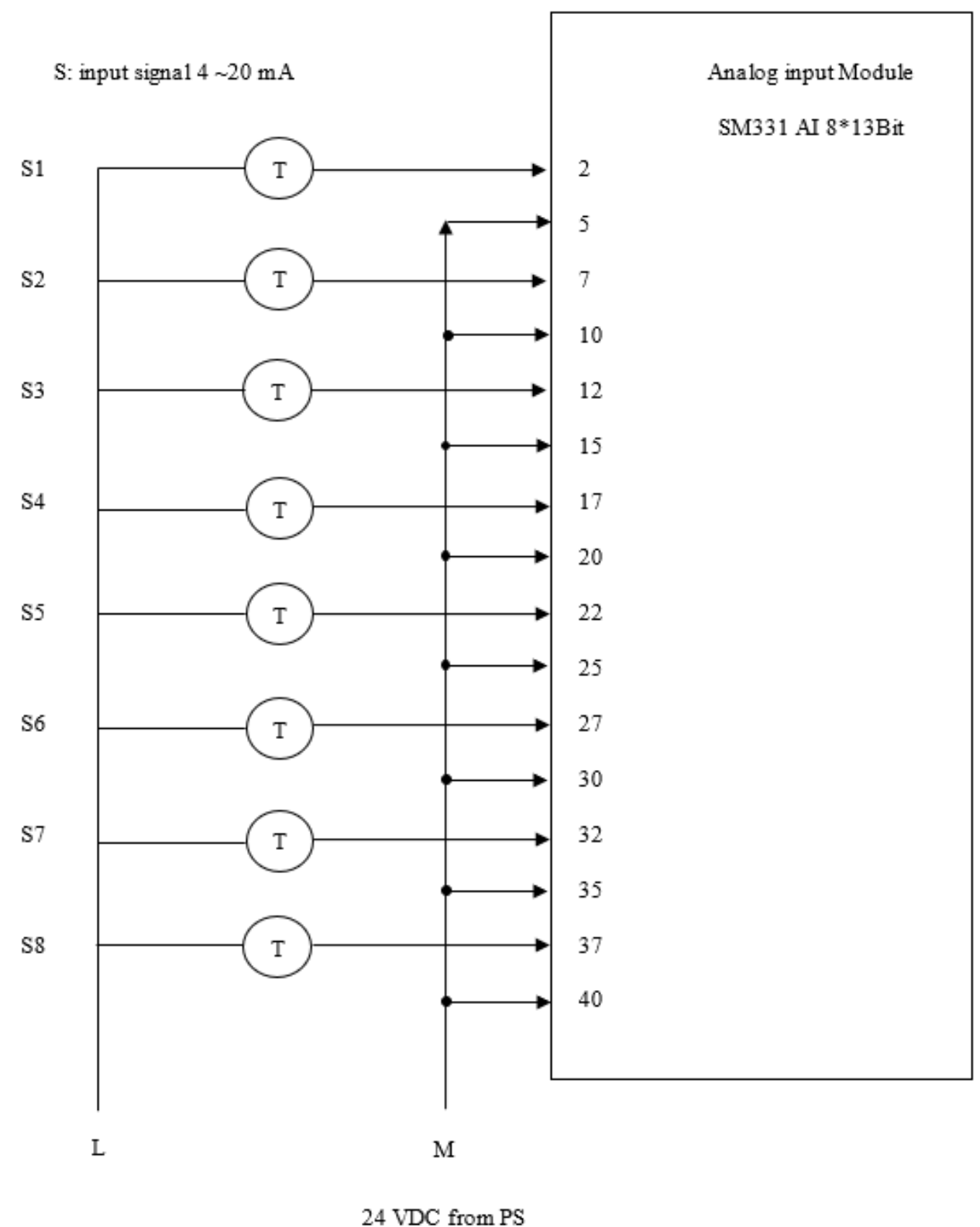

Fig 5. Analog input module, $\mathrm{M}=$ negative, $\mathrm{L}=$ positive, and $\mathrm{T}=$ Transmitter. 
DATA (D)

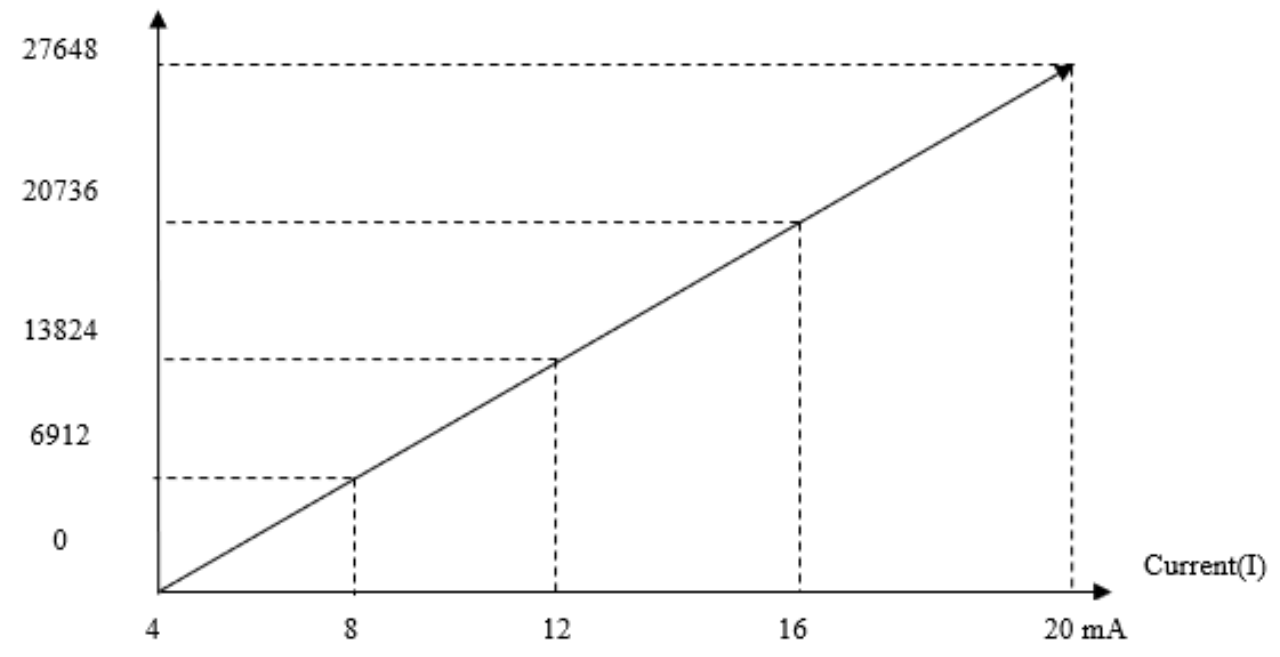

Fig 6. Current-DATA relation. 


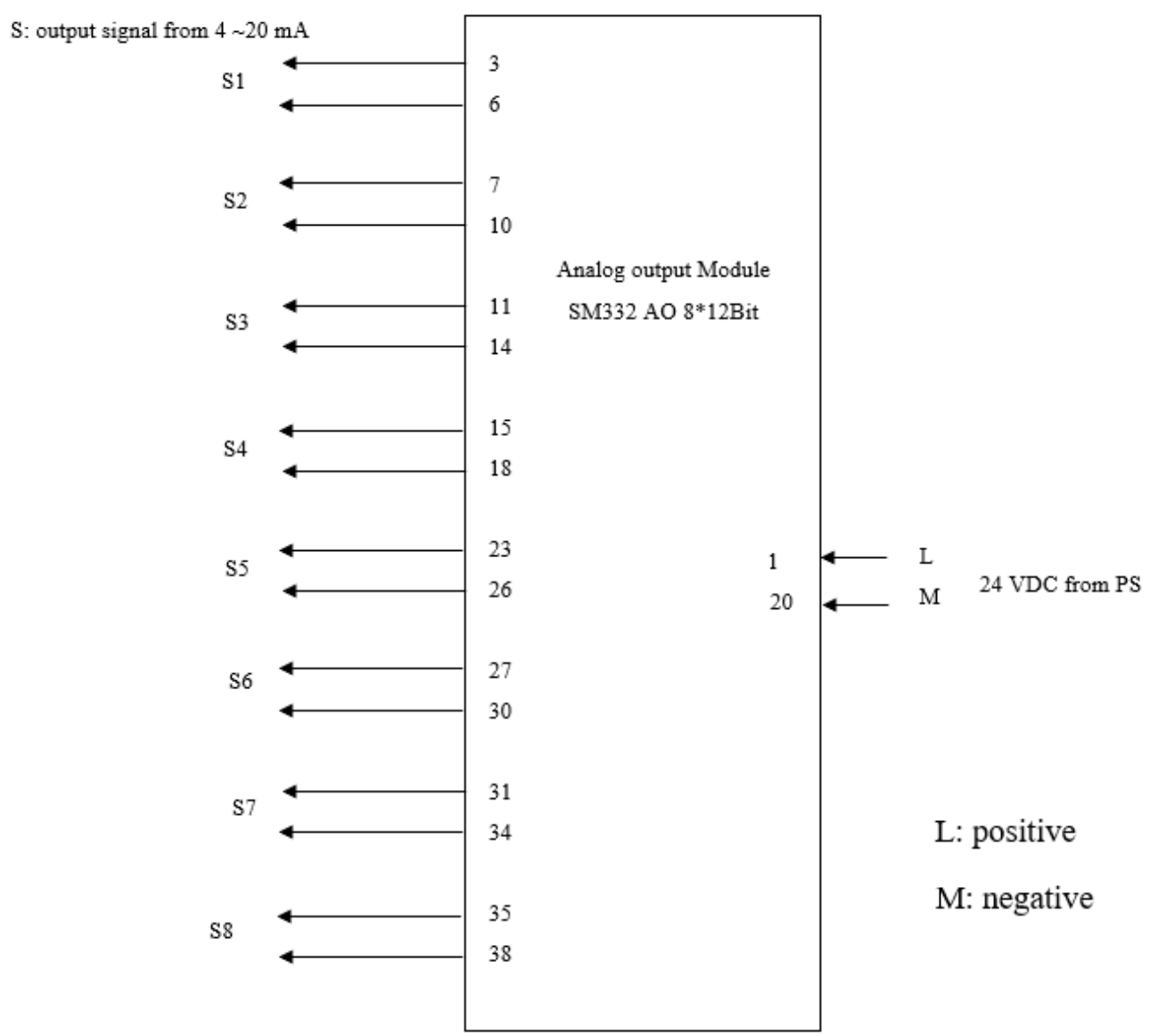

Fig 7. Analog output module.

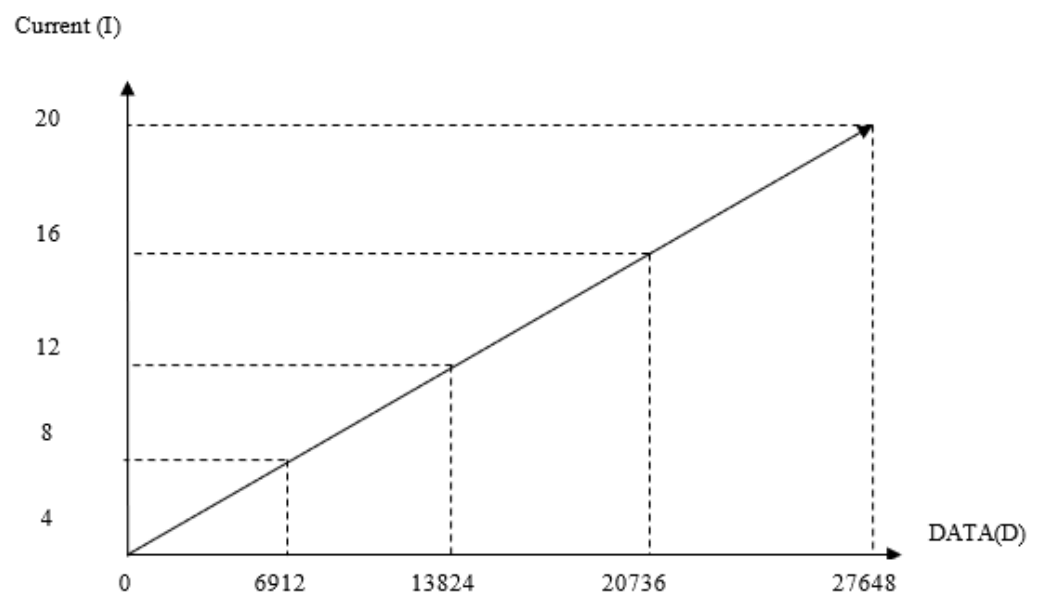

Fig 8. DATA-Current relation. 


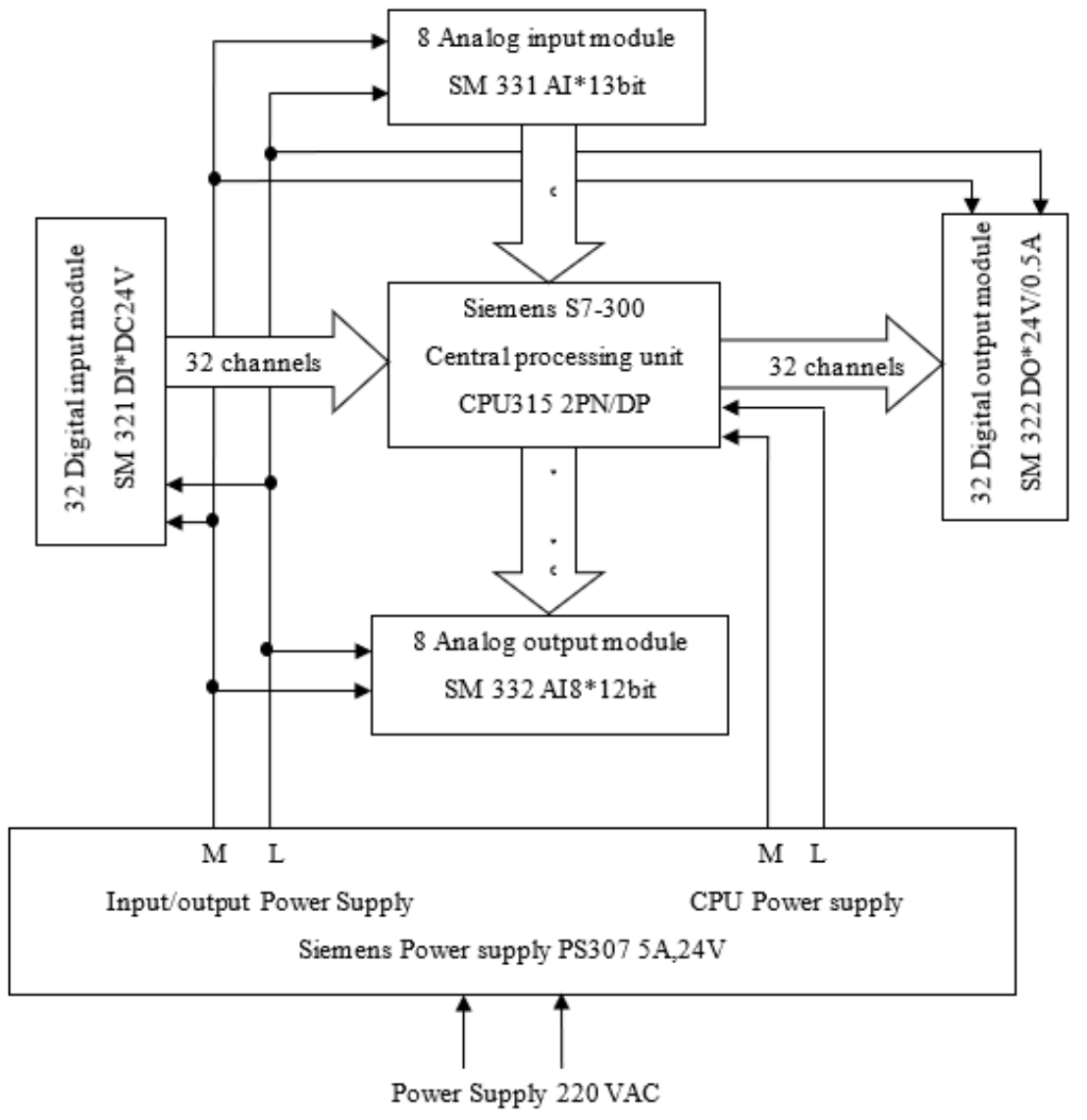

Fig 9. Block diagram of the S7-300.
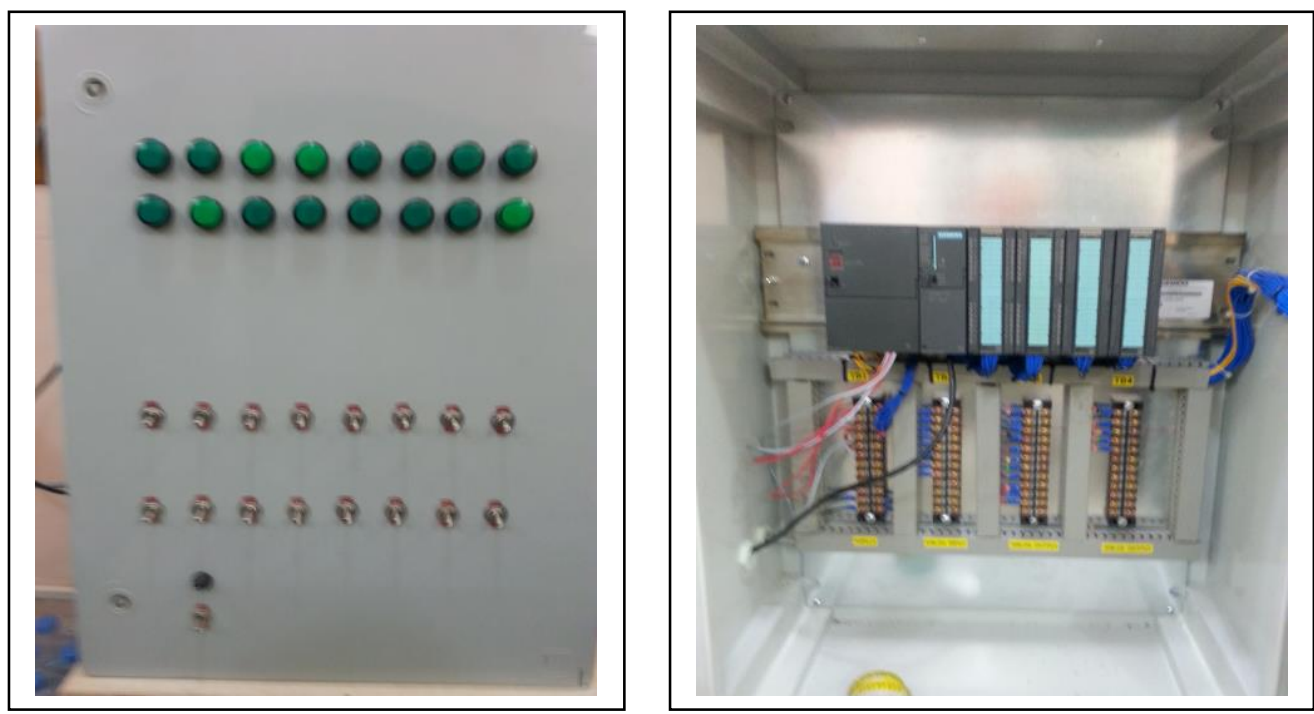

Fig 10. photograph of the Siemens S7-300 cabinet front. Fig 11. photograph of the Siemens S7-300 cabinet 


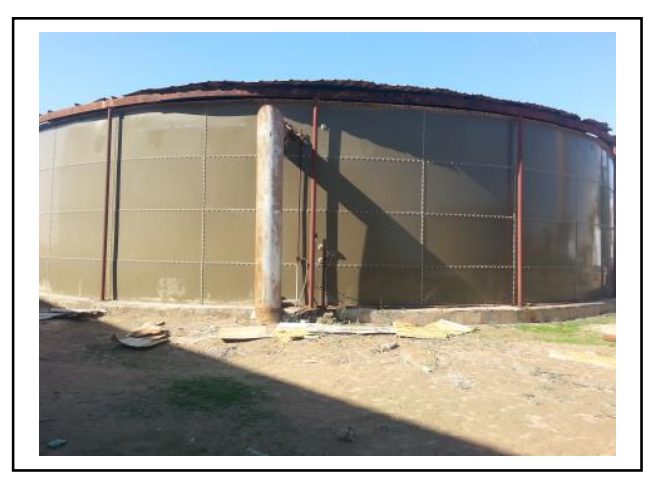

Fig 12. water vessel (V-604) in the water

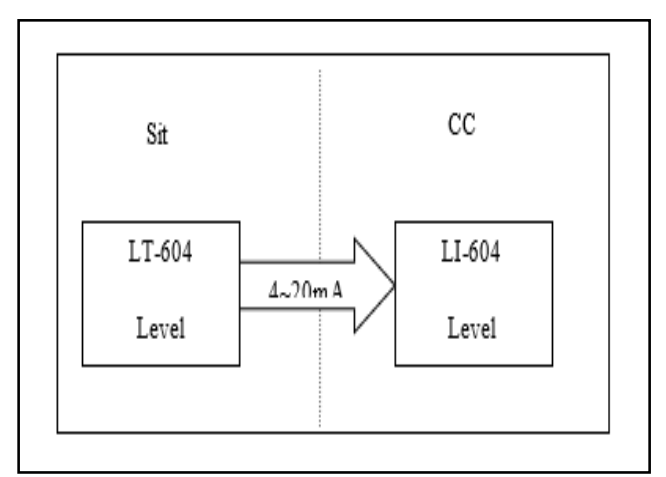

Fig 13. Block diagram of the old level measuring and monitoring system.

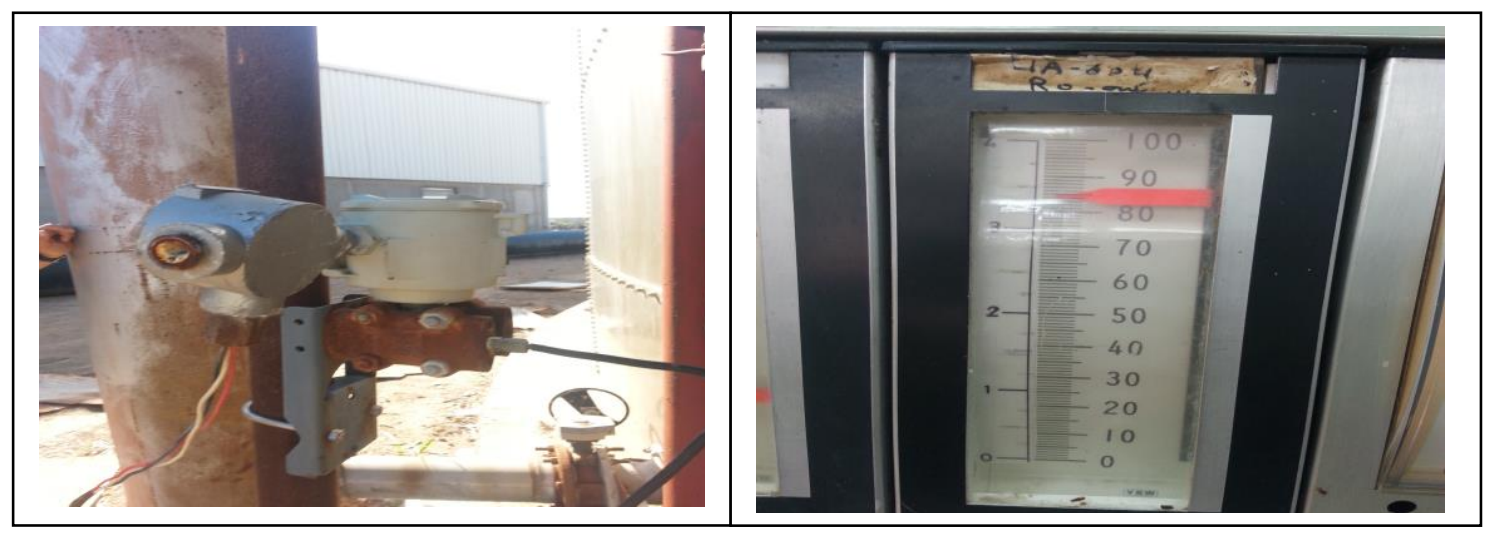

Fig 14. level transmitter (LT-604) in the water treatment unit.

Fig 15. level indicator (LI-604) in the water treatment unit.

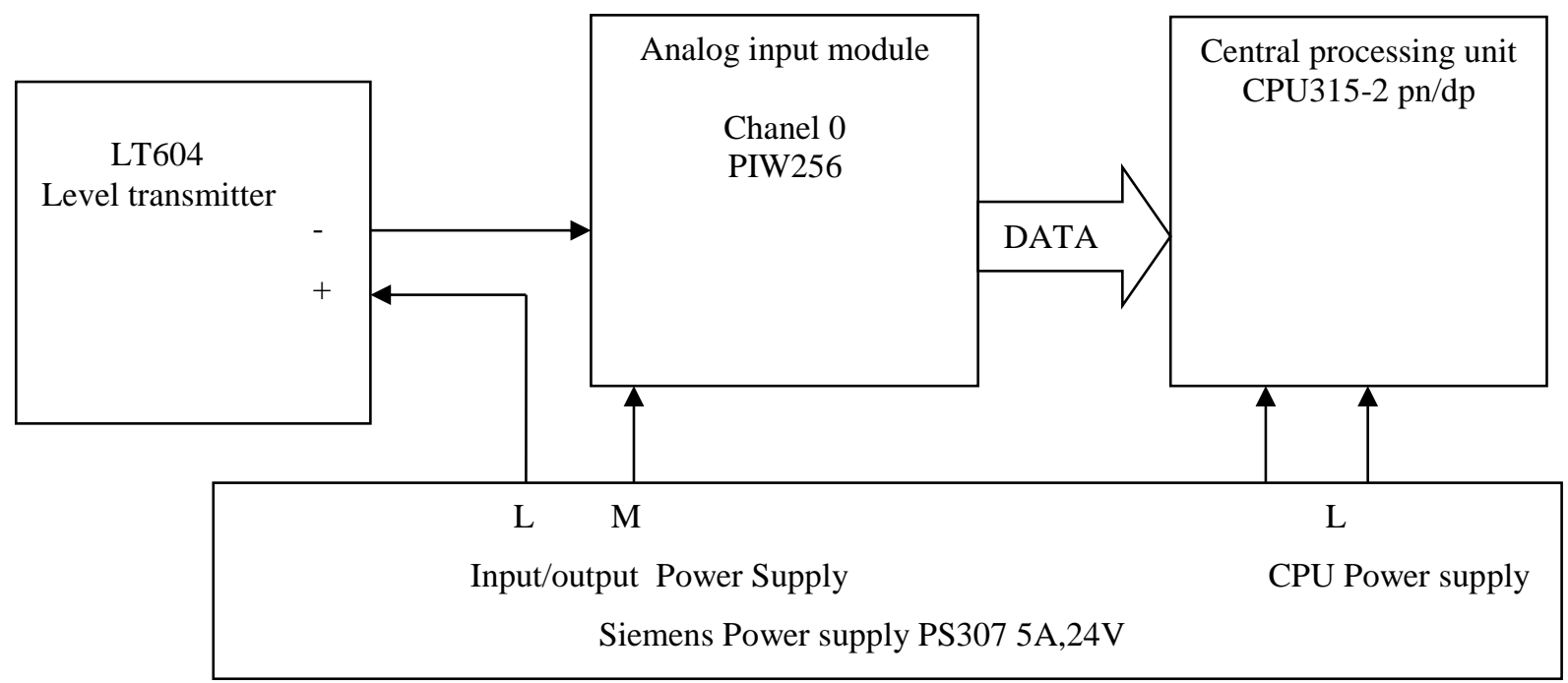

Fig 16. Block diagram of the new measuring and monitoring system 


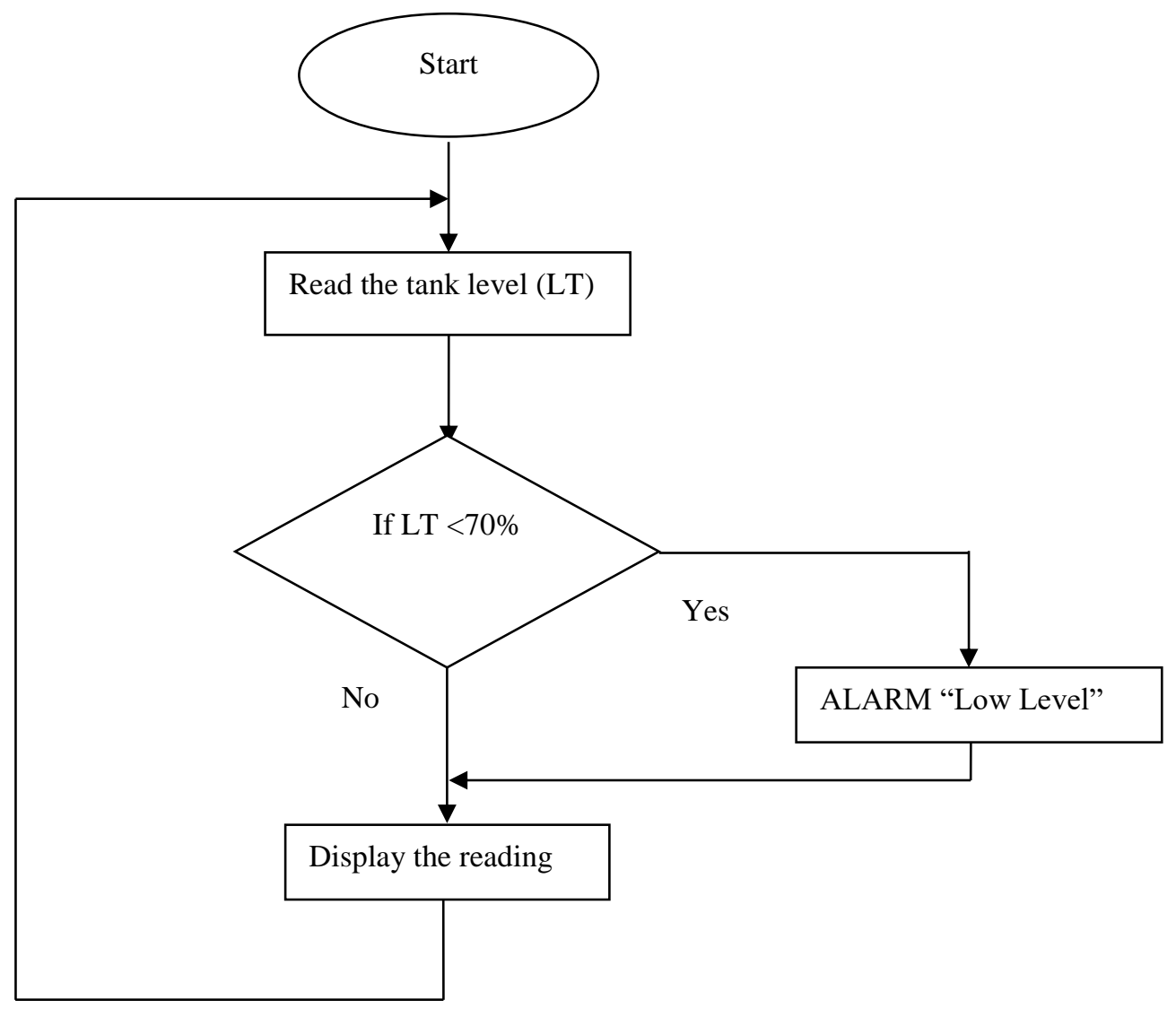

Fig 17. Level Measurement flow chart

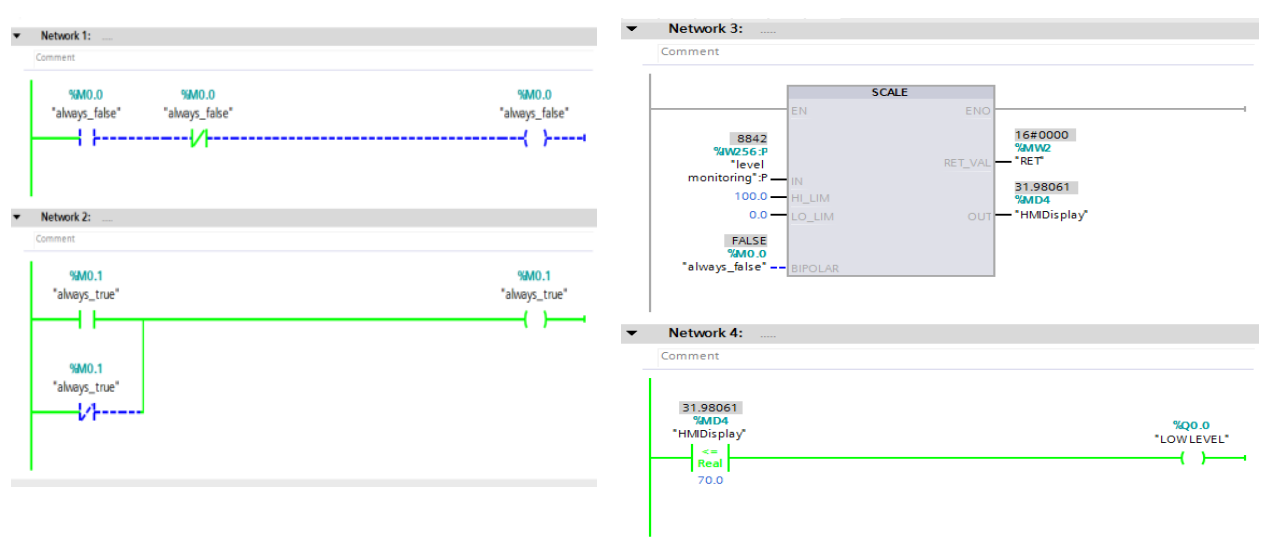

Fig 18. Ladder diagram of the Level Measurement. 


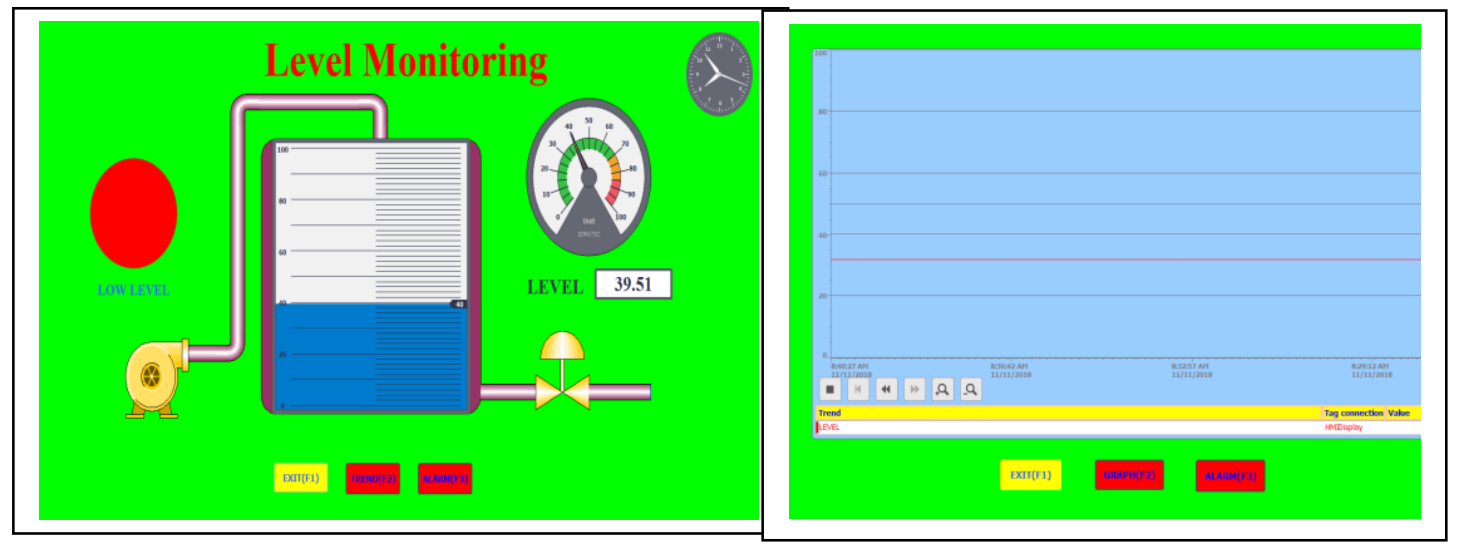

Fig 19. Graphic page of the Level Measurement

Fig 20. Trend page of the Level Measurement.
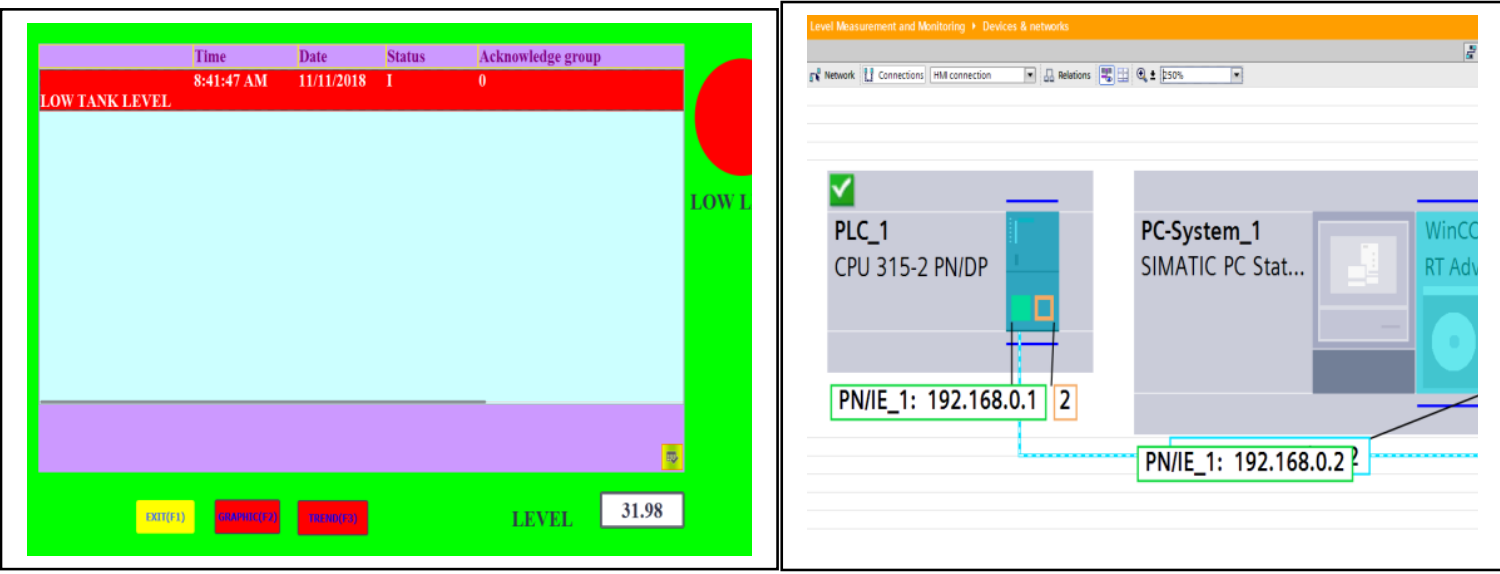

Fig 21. Alarm page of the Level Measurement.

Fig 22. Profinet Topology of the Level Measurement.

Table 1. Analog input limits.

\begin{tabular}{|c|c|c|c|c|}
\hline Index & Ratio & Input range $(\mathrm{mA})$ & Value in HEX & Value in DEC \\
\hline 1 & $0 \%$ & 4 & 0 & 0 \\
\hline 2 & $25 \%$ & 8 & $1 \mathrm{~B} 00$ & 6912 \\
\hline 3 & $50 \%$ & 12 & 3600 & 13824 \\
\hline 4 & $75 \%$ & 16 & 5100 & 20736 \\
\hline 5 & $100 \%$ & 20 & $6 \mathrm{C} 00$ & 27648 \\
\hline
\end{tabular}

Table 2. Analog output limits.

\begin{tabular}{|c|c|c|c|c|}
\hline Index & Ratio & Value in DEC & Value in HEX & Output range (mA) \\
\hline 1 & $0 \%$ & 0 & 0 & 4 \\
\hline 2 & $25 \%$ & 6912 & $1 \mathrm{~B} 00$ & 8 \\
\hline
\end{tabular}




\begin{tabular}{|l|c|c|c|c|}
\hline 3 & $50 \%$ & 13824 & 3600 & 12 \\
\hline 4 & $75 \%$ & 20736 & 5100 & 16 \\
\hline 5 & $100 \%$ & 27648 & $6 C 00$ & 20 \\
\hline
\end{tabular}

Table 3. S7-300 modules arrangement inside the rail.

\begin{tabular}{|c|c|c|c|c|c|}
\hline Slot 0 & Slot 1 & Slot 2 & Slot 3 & Slot 4 & Slot 5 \\
\hline Power supply & CPU & Digital input & Digital output & Analog input & Analog output \\
\hline
\end{tabular}

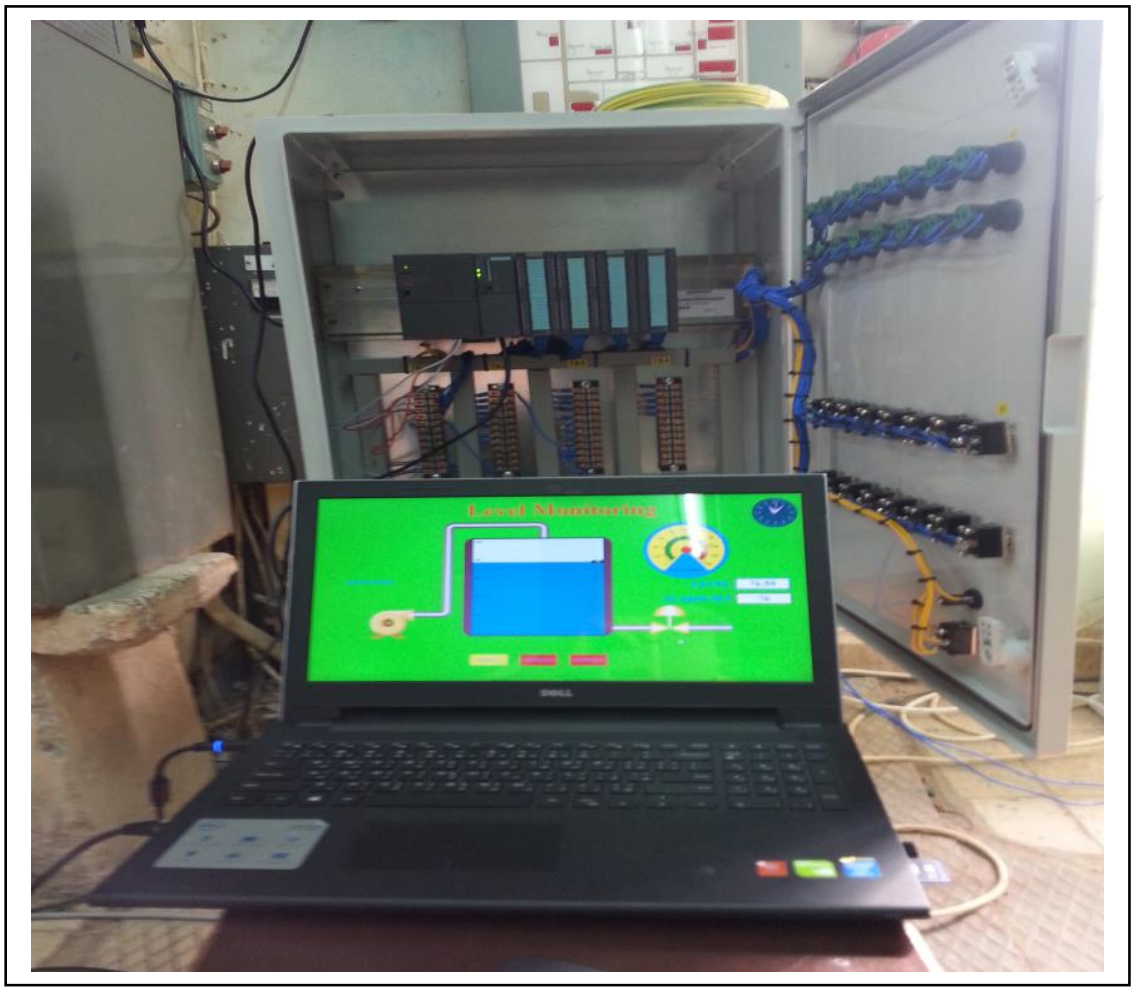

Fig 23. Photograph of overall measurement and monitoring system.

\section{Results and Discussion}

In this present study, Siemen's control cabinet-based S7-300 PLC has been designed and implemented. Several Siemens modules were used to build the cabinet such as power supply, CPU, DI, DO, AI and AO. The latest software of Siemens is the TIA. The TIA was used to program the sequence of the process as a LADDER diagram in PLC and to design the HMI pages in the PC to fabricate the SCADA system. The cabinet has been tested in the water treatment unit of the factory for commissioning. Two-wire differential pressure transmitters were connected to the cabinet to measure the vessel level and to display the value on HMI pages. The results obtained that the practical level values are identical to the theoretical values and according to the equation of current-DATA relation. We found deviation in the reading of the 
old monitoring system, but the reading of the new monitoring system was very accurate. Also, the HMI software gave the expected findings and we found that the new monitoring system is better than the old monitoring system. The experimental tests of the PLC set and HMI software are successfully done. The cabinet can be used as training and a research kit and many types of research in automation can be done by using this kit. We found that using of SCADA system instead of a classic system has several advantages such as reduce time consumption, reduce human resource consumption, Increase production, greater accessibility (remotely), Instantaneous alert on alarms or events, organized data records and report generation automatically, data analysis (real-time and historical data plotting of graphs, charts, trends, etc.), easy user interfaces for interaction (graphical HMI interface with animation for easy understanding), Automated calculation and easy of diagnosis and maintenance.

\section{References}

[1] P. Logic et al., "Chapter 8 : Programmable Logic Controller ( PLC )," Ind. Electron. Control, vol. 485 , no. 8 , p. $1,2106$.

[2] V. M. Kantilal and A. P. Lakhlani, "ZIGBEE based Wireless Monitoring and Controlling of Automation System using PLC \& SCADA,” Int. J. Adv. Res. Electr. Electron. Instrum. Eng., vol. 3, no. 1, pp. 6768-6775, 2014.

[3] D.Baladhandabany, S.Gowtham, T.Kowsikkumar, and P.Gomathi, "PLC Based Automatic Liquid Filling System,” Int. J. Comput. Sci. Mob. Comput., vol. 4, no. 3, pp. 684-692, 2015.

[4] Y. N. Burali, "PLC Based Industrial Crane Automation \& Monitoring,” Int. J. Eng. Sci., vol. 1, no. 3, pp. 01-04, 2012.

[5] E. Yilmaz and S. Katrancioglu, "Designing programmable logic controller (PLC) experiment set with internal experiment blocks,” Procedia - Soc. Behav. Sci., vol. 28, pp. 494-498, 2011, doi: 10.1016/j.sbspro.2011.11.095.

[6] N. Thakur and M. Hooda, "A Review Paper on Digital Watermarking Techniques \& Its Applications," Int. J. Sci. Res., vol. 5, no. 6, pp. 1864-1868, 2016, doi: 10.21275/v5i6.nov164699.

[7] V. M. Kumar, A. Sivasankar, G. Prabakaran, R. Dhanabal, and A. M. Krishnan, "Design and Implementation of PLC Based Control Panel for Hydraulic Assembly Press,” pp. 1704-1711, 2017, doi: 10.15662/IJAREEIE.2017.0603097.

[8] P. Chakraborty, A. Hossain, and V. Pillarisetty, "Designing and assembling of a programmable logiccontrols (PLC) laboratory trainer and advanced research setup,” ASEE Annu. Conf. Expo. Conf. Proc., vol. 2016-June, 2016, doi: 10.18260/p.26701.

[9] A. Thesis, "PLC Based Implementation of Solar Photovoltaic Energy Maximum Power Point Tracking Techniques," no. October, 2014.

[10] A. Thesis and S. To, "Dedicated to my parents, sisters, dear wife and lovely sons Arshed azeez saher," no. July, 2017. 
[11] M. J.Marie, G. A. Al-Suhail, and H. T. Khadim, "Developing Al-Najaf Cement Plant using Wireless SCADA System,” Int. J. Comput. Appl., vol. 64, no. 13, pp. 17-23, 2013, doi: 10.5120/10693-5602.

[12] V. M. Koshti and S. M. Joshi, "Design of Human Machine Interface for Plc Based Automation System,” IFAC Proc. Vol., vol. 40, no. 18, pp. 343-346, 2007, doi: 10.3182/20070927-4-ro3905.00057.

[13] A. M. Anusha and A. V Sutagundar, "Design of a PLC Based Elevator Control System," vol. 6, no. 2, pp. 32-36, 2015.

[14] L. Chitra, S. Siranjeevi, A. Manikandan, and G. Saravanan, "Automation of Batch Production in Paint Industry using PLC,” Int. J. Innov. Technol. Explor. Eng., vol. 9, no. 2S4, pp. 435-440, 2019, doi: 10.35940/ijitee.b1207.1292s419.

[15] V. T. Samuel, "Control of Smart Building Using Advanced SCADA,", THESIS,University of South Florida, January, 2013. 